\title{
Binge Eating Disorder and the Outcome of Bariatric Surgery at One Year: A Prospective, Observational Study
}

\author{
Thomas A. Wadden, Ph.D. ${ }^{1}$, Lucy F. Faulconbridge, Ph.D. ${ }^{1}$, LaShanda R. Jones-Corneille, \\ Ph.D. ${ }^{1}$, David B. Sarwer, Ph.D. ${ }^{1}{ }^{1}$, Anthony N. Fabricatore, Ph.D. ${ }^{1}$, J. Graham Thomas, \\ Ph.D. ${ }^{3}$, G. Terence Wilson, Ph.D. ${ }^{4}$, Madeline G. Alexander, Ph.D. ${ }^{1}$, Melissa E. Pulcini, B.A. ${ }^{1}$, \\ Victoria L. Webb, B.A. ${ }^{1}$, and Noel N. Williams, M.D. ${ }^{2}$ \\ ${ }^{1}$ University of Pennsylvania School of Medicine, Department of Psychiatry \\ ${ }^{2}$ University of Pennsylvania School of Medicine, Department of Surgery \\ ${ }^{3}$ Warren Alpert School of Medicine at Brown University, Department of Psychiatry and Human \\ Behavior \\ ${ }^{4}$ Rutgers -The State University of New Jersey, Graduate School of Applied and Professional \\ Psychology
}

\begin{abstract}
Previous studies have suggested that binge eating disorder (BED) impairs weight loss following bariatric surgery, leading some investigators to recommend that patients receive behavioral treatment for this condition before surgery. However, many of these investigations had significant methodological limitations. The present observational study used a modified intent-to-treat population to compare 1-year changes in weight in 59 surgically-treated participants, determined preoperatively to be free of a current eating disorder, with changes in 36 individuals judged to have BED. Changes in weight and binge eating in the latter group were compared with those in 49 obese individuals with BED who sought lifestyle modification for weight loss. BED was assessed using criteria proposed for the Diagnostic and Statistical Manual (DSM) 5. At 1 year, surgicallytreated participants without BED lost $24.2 \%$ of initial weight, compared with $22.1 \%$ for those with BED ( $>>0.309)$. Both groups achieved clinically significant improvements in several cardiovascular disease (CVD) risk factors. Participants with BED who received lifestyle modification lost $10.3 \%$ at 1 year, significantly $(\mathrm{p}<0.001)$ less than surgically-treated BED participants. The mean number of binge eating days (in the prior 28 days) fell sharply in both BED groups at 1 year. These two groups did not differ significantly in BED remission rates or in improvements in CVD risk factors. The present results, obtained in carefully studied participants, indicate that the preoperative presence of BED does not attenuate weight loss or improvements in CVD risk factors at 1 year in surgically treated patients. Longer follow-up of participants is required.
\end{abstract}

\section{Keywords}

Binge eating; obesity surgery; eating disorders; lifestyle modification

All correspondence to: Thomas Wadden, Ph.D., University of Pennsylvania, 3535 Market Street, Suite 3108 (3 ${ }^{\text {rd }}$ floor), Philadelphia, PA 19104. Tel.: 215-898-7314; Fax: 215-898-2878; wadden@mail.med.upenn.edu.

Disclosure

David Sarwer serves as a consultant for Allergan, Baronova, Enteromedics, and Ethicon Endo-Surgery. He also sits on the Board of Directors for the Surgical Review Corporation. Anthony Fabricatore serves as a consultant to Allergan. The other authors have no conflicts of interest to declare. 
Binge eating disorder presents a clinical challenge for surgeons and mental health practitioners who assess patients with extreme obesity to determine whether they will benefit from bariatric surgery. This disorder is characterized by consuming an objectively large amount of food in a discrete period of time (i.e., 2 hours), with an accompanying sense of loss of control and subsequent distress about the overeating $(1,2)$. It also is associated with an increased risk of depression, anxiety, and other psychopathology (3-6). Estimates of the prevalence of binge eating disorder (BED) in surgery candidates range from 5\% to 50\% (7-12). Self-report questionnaires overestimate the prevalence of this disorder (10), which typically is diagnosed in approximately $5 \%$ to $25 \%$ of surgery candidates when assessed using a structured clinical interview and strict criteria (7-10).

Several investigators (13-15) have reported that BED is associated with impaired weight loss following surgery, leading some (16) to recommend that patients with this condition receive pre-operative behavioral counseling. Other studies have found no relationship between the preoperative presence of BED and post-surgical weight loss (17-21). The contradictory findings are likely attributable to several factors that include differences in the assessment of preoperative BED status which, in many cases, was determined using selfreport questionnaires or retrospective recall, each of which is sub-optimal. Other investigators have concluded that postoperative loss of control over eating, regardless of its presence pre-operatively, is associated with suboptimal weight loss (20-23). These conflicting findings have left surgeons and mental health professionals uncertain about how best to treat extremely obese patients with BED. Should these patients routinely be referred for preoperative behavioral counseling (16)? Alternatively, should practitioners assume that BED will remit following surgery, as observed in several studies $(17,24,25)$, and that these patients will achieve satisfactory weight loss and improvements in co-morbid conditions? Expert guidelines do not consider BED to be an absolute contraindication to surgery (26), but practitioners are advised to assess the severity and consequences of the condition both pre- and post-operatively $(26,27)$.

The present observational study was designed to determine whether bariatric surgery candidates who were diagnosed with BED, using the Eating Disorder Examination (28), would lose significantly less weight 1 -year post-surgery than would patients who were free of this condition. To achieve maximal possible differences between groups, the non-BED participants were determined to be free of a current eating disorder. In addition, the occurrence of binge eating was assessed in both groups over the course of the year to determine the effects of bariatric surgery on the remission (or precipitation) of this behavior. In designing the study, we included a non-randomly selected comparison group of individuals who had BED and met BMI criteria for bariatric surgery but, instead, sought treatment by a comprehensive program of lifestyle modification. This group potentially provides estimates of changes in weight and eating behavior that bariatric surgery candidates with BED could be expected to achieve if they could not obtain surgery and instead were referred to a behavioral weight loss program. We decided against trying to conduct a randomized controlled trial of this topic because of potential ethical and practical issues in assigning participants to treatments.

\section{Method}

\section{Bariatric Surgery Participants}

Surgery participants were recruited from the Bariatric Surgery Program at the Hospital of the University of Pennsylvania (from September 2005 to December 2008). All candidates must complete several pre-operative evaluations, including a behavioral assessment, to determine that they are free of contraindications to surgery $(26,29)$. The behavioral assessment includes the Beck Depression Inventory-II (BDI-II) (30), the Weight and 
Lifestyle Inventory (WALI) (31), and a clinical interview which is conducted by mental health professionals at the Center for Weight and Eating Disorders (29). The present study was approved by the Institutional Review Board at the University of Pennsylvania.

After completing the behavioral assessment, mental health professionals (i.e., clinicians) summarized their clinical findings for the candidates, answered any questions, and indicated that they would forward a letter to the referring surgeon, which reviewed the findings of the evaluation. Clinicians then informed select patients that the Center was conducting a research study to compare changes in weight, eating behavior, and other outcomes in surgical candidates with and without BED. Clinicians were instructed to share this information with all surgery candidates who appeared to potentially meet criteria for BED, based on patients' responses to the Questionnaire on Eating and Weight Patterns (QEWP) (32), which is included in the WALI. The information also was shared with individuals who appeared to be free of a current eating disorder. Candidates who wished to learn more about the study were directed to the study's research coordinator.

Research coordinators informed potential participants of the nature and requirements of the study, answered any questions, and obtained written informed consent from interested individuals. Consented participants then completed the Eating Disorder Examination (EDE), which was administered by trained assessors who were masked to the clinicians' provisional diagnoses. The EDE uses a structured clinical interview to assess participants' eating-related attitudes and behaviors and yields diagnoses for the major eating disorders. The EDE has excellent reliability when administered by trained examiners (33). Wilson et al recently examined the agreement between assessors in determining the number of episodes of binge eating, as well as days on which binge eating was present, and reported interrater reliability coefficients of 0.97 for both measures (34).

The present study used an abbreviated version of the EDE to assess the occurrence of objective binge eating episodes. Such episodes refer to eating an objectively large amount of food (as judged by the examiner) in a discrete period of time, with the patients' report of loss of control during the episode. (The amount of food eaten must be definitely larger than most people would eat during a similar period of time under similar circumstances.) Patients also must report distress about the overeating episode. We also assessed subjective binge episodes, which are characterized by a sense of loss of control but without the consumption of an objectively large amount of food. At screening only, participants also were evaluated for objective overeating episodes, which involve the consumption of an objectively large amount of food but without the experience of loss of control or distress. (An example would be eating a whole pizza and reporting enjoyment of the eating, with no loss of control.) The EDE initially queries participants about their eating behavior for the previous 28 days, which can be extended to the previous 3 to 6 months to obtain information needed for the diagnosis of different eating disorders.

For this study, participants were diagnosed with BED if they reported at least one objective binge eating episode per week for the past 3 months, met associated behavioral criteria (including distress about their eating), and were free of symptoms of bulimia nervosa. These are the frequency and duration criteria proposed for DSM-5 for the diagnosis of BED (35), and were selected at the outset of the study. The proposed frequency criteria are based on findings of no significant differences in psychopathology, eating-specific concerns, or treatment outcomes in persons who engage in binge eating only once a week versus two or more times weekly $(19,36-38)$. (We note that current DSM-IV criteria classify persons who binge eat only once per week as having subclinical BED.) 
Participants who reported no objective binge eating episodes and not more than one objective overeating episode per week during the previous 3 months (and were free of symptoms of bulimia nervosa) were considered to be free of a current eating disorder. These individuals were selected as a non-BED comparison group and were recruited consecutively, with periodic breaks in recruitment to track recruitment of BED patients. Individuals with other degrees of eating pathology were excluded from the study.

\section{Surgical intervention}

Participants, in consultation with their surgeon, elected to undergo Roux-en-Y gastric bypass (RYGB) or adjustable gastric banding (AGB), each of which was conducted laparoscopically (unless complications prevented this), following standard methods $(39,40)$. The great majority of both operations were performed by the last author (NNW), with a minority conducted by surgeons whom he had trained, thus, providing uniformity of approach. The research team (excepting NNW) was not responsible for evaluating applicants' medical eligibility to undergo surgery, a determination made by the Bariatric Surgery Program's medical consultants. Similarly, the research team (excepting NNW) was not responsible for any aspects of participants' medical care following surgery.

\section{Lifestyle Modification Participants}

Participants in the lifestyle modification group were recruited using advertisements for a weight loss program for obese men and women who suffered from binge eating. Similar to the surgery study, participation was open to individuals who were at least 18 years of age and had a BMI $\geq 40 \mathrm{~kg} / \mathrm{m}^{2}$ (or $\geq 35 \mathrm{~kg} / \mathrm{m}^{2}$ in the presence of a co-morbid condition). The research team was responsible for the initial medical evaluation (and monitoring during treatment) of participants in the lifestyle modification group and followed exclusion criteria used in prior studies $(41,42)$. These included: a history of cerebrovascular, cardiovascular, kidney, or liver disease; type 1 diabetes; uncontrolled hypertension $(\geq 140 / 90 \mathrm{~mm} \mathrm{Hg})$; pregnancy or lactation; the use of medications known to affect body weight (i.e., steroids); and a weight loss $\geq 5 \%$ of initial weight (or use of anorectic agents) in the prior 6 months. Participants with a BDI score $>28$, indicative of severe symptoms of depression, were excluded, except in cases in which depression appeared to be related principally to distress about body weight. The use of antidepressant medications was permitted. (These were the same general guidelines used to evaluate candidates' appropriateness for surgery.)

Respondents to advertisements completed a telephone screening. Those who appeared to meet criteria were scheduled for an interview with a psychologist who informed participants of the nature and requirements of the study and obtained their written, informed consent to participate. As with surgery applicants, the psychologist reviewed participants' responses to the QEWP to assess whether they appeared to meet criteria for BED. However, this latter determination was left to masked assessors, who administered the EDE to lifestyle modification applicants, following the same procedures used in selecting surgical participants with BED.

Lifestyle modification intervention-Participants were provided a comprehensive group program of lifestyle modification that included weekly sessions from weeks 1 to 20 , bi-weekly meetings from weeks 22 to 40 , and monthly sessions through week 52 . Sessions lasted 90 minutes, included 7 to 10 participants, and were led by a clinical psychologist, following a protocol described previously (43). At Week 1, participants recorded their food intake in diaries provided and at Week 2 were instructed to consume a self-selected diet (of conventional foods) of 1800-2000 kcal/d. From Weeks 3-14, they were prescribed a portion-controlled diet that combined four servings a day of a liquid meal replacement (HMR 800; Boston, MA) with an evening meal of a frozen food entrée, a cup of garden 
salad, and a serving of fruit. Liquid meal replacements were given to participants free of charge, and each serving provided $160-170 \mathrm{kcal}$, with $14 \mathrm{~g}$ of protein. Participants purchased frozen food entrees, with instructions to select items that provided approximately $250-300 \mathrm{kcal}$ and 20 to $25 \mathrm{~g}$ of protein. Participants were instructed to consume additional servings of fruits and vegetables to achieve a total intake of 1200-1300 kcal/d. Beginning at Week 14, participants decreased their consumption of liquid meal replacements with the goal, by Week 18, of consuming a diet of conventional foods of $1400-1600 \mathrm{kcal} / \mathrm{d}$.

Participants were provided one liquid meal replacement a day for the duration of the 1-year study to facilitate maintenance of lost weight $(44,45)$.

The lifestyle modification curriculum included behavioral techniques described previously (43). Participants were encouraged to gradually increase their physical activity to 180 minutes per week of aerobic activity (principally walking). Cognitive therapy for BED, as described elsewhere $(34,46)$, was not provided because we wished to assess the effects of a traditional weight control program on binge eating.

\section{Outcome Measures}

Weight—Body weight was measured on a digital scale (Detecto, model 6800A) while participants were dressed in light clothing, without shoes. Weight was assessed at the screening visit and at a subsequent baseline visit, scheduled approximately 1 to 2 weeks before surgery participants underwent their procedures and 1 week before Lifestyle Modification participants began group treatment. Weight was re-measured at 2, 6, and 12 months post-baseline. Height was measured at baseline using a wall-mounted stadiometer.

Binge eating-Binge eating was assessed at the screening visit and months 2, 6, and 12 using the EDE (28). On each occasion, the examiner determined the number of objective (and subjective) binge eating episodes participants had experienced in the prior 28 days. Examiners also counted the number of days on which binge episodes were present, an assessment that eliminates ambiguity about whether a prolonged objective binge episode (e.g., all afternoon) counts as one episode or potentially as several.

Cardiovascular disease (CVD) risk-Fasting blood samples were obtained at baseline and months 2, 6, and 12, as described previously (41), and analyzed for CVD risk factors that included high sensitivity $\mathrm{C}$ reactive protein (hs-CRP). Glucose and insulin also were assessed, and insulin sensitivity was estimated using the homeostasis model of insulin sensitivity (HOMA). All analyses were conducted by Quest Diagnostics (Lynhurst, NJ).

\section{Statistical Analyses}

Data were examined for a modified intent-to-treat (ITT) population that included all participants who received treatment (i.e., underwent surgery or attended at least one lifestyle modification session) and provided at least one post-baseline measurement of body weight. Participants without a post-baseline measurement of weight were excluded because of the lack of adequate estimates of weight change (needed for imputation) in surgically-treated patients who do not participate in post-surgical follow-up. For the modified ITT population, differences in baseline characteristics between surgery patients, with and without BED, were analyzed using independent groups t-tests and chi-square tests, as were differences between participants with BED who received surgery or lifestyle modification. The study's primary outcome was percentage change in initial weight at month 12 in surgically-treated participants with and without BED. A two-tailed $P$ value of 0.05 was set for this primary comparison. These two groups also were compared on changes in CVD risk factors, with a two-tailed $\mathrm{P}$ value $\leq 0.01$ required for any difference to be considered statistically significant (to control for multiple comparisons). The study's main secondary outcomes 
were change in weight and binge eating days at month 12 in participants with BED who were treated by surgery versus lifestyle modification. A two-tailed $P$ value of 0.05 was set for the separate tests of weight and binge eating. These two groups also were compared on changes in CVD risk factors (with P set at $\leq 0.01$ for all comparisons), as well as differences in the remission of BED (i.e., no longer meeting criteria for BED for the prior 3 months).

Changes in weight, binge eating days, and CVD risk factors were examined using linear mixed-effects models, which controlled for initial BMI, type of surgery (i.e., RYGB vs AGB), age, gender, ethnicity, presence of type 2 diabetes, and the baseline value of the outcome. As a first step, unconditional models were used to determine whether a linear or quadratic trend best fit the longitudinal trajectory of the outcomes, and whether slopes should be treated as random effects. In a second step, covariates were added to the conditional model in order to account for variability in the trajectory of the outcomes. Continuous covariates were centered using the grand mean prior to analysis. Differences between groups in the remission of BED were determined using Fisher's exact test. For this analysis only, data from the nearest previous assessment point were carried forward in cases of missing data, as performed in a prior study (34).

\section{Results}

\section{Participant Enrollment and Retention}

As shown in Figure 1, 62 surgery candidates were identified who consented to participate in the study and met criteria for BED, of whom 51 ultimately had surgery. (Eleven participants either were denied insurance coverage or decided against surgery.) Of these 51, 36 (70.6\%) returned for at least one post-operative (i.e., post-baseline) assessment of weight, with 30 $(58.8 \%)$ returning at month 12 . A total of 89 candidates were identified who were currently free of an eating disorder (i.e., non-BED participants), of whom 80 ultimately had surgery. (Nine were denied insurance coverage or decided against surgery.) Of these 80, 59 (73.8\%) returned for at least one post-baseline assessment, with $51(63.8 \%)$ returning at month 12. For the Lifestyle Modification participants, a total of 82 individuals completed an initial onsite evaluation, of whom 57 met inclusion/exclusion criteria. Fifty-one participants enrolled in the study, $49(96.1 \%)$ provided a post-baseline measurement of body weight, and 42 (82.4\%) completed the month 12 assessment. A significantly $(\mathrm{P}=0.011)$ greater percentage of participants in the Lifestyle-BED group (82.4\%) attended the 12-month assessment, compared with the Surgery-BED (58.8\%) and Surgery-nonBED participants (63.8\%). The latter two groups did not differ significantly from each other. Analyses which collapsed across the three treatment groups included in the modified ITT population showed that the 21 (of 144) participants who did not complete the 12-month assessment were significantly younger than those who did complete it $(40.8 \pm 2.1$ vs $45.3 \pm 0.9 \mathrm{yr} ; \mathrm{P}=0.045)$, with no significant differences in completion rates related to BMI, gender, education or race/ ethnicity. Similarly, the 38 (of 182) participants who failed to provide a post-baseline measurement of weight (thus excluding them from the analyses) were significantly younger than those who provided a measurement $(40.8 \pm 1.6$ vs $44.6 \pm 0.8 \mathrm{yr} ; \mathrm{P}=0.031)$, with no other significant differences between groups on the other demographic variables examined.

\section{Participants' Baseline Characteristics}

Table 1 presents baseline characteristics of participants in the three groups, based upon the modified ITT population. The two surgery groups, without and with BED, were very similar on demographic and other baseline variables including age (43.8 \pm 1.3 vs $47.0 \pm 1.6 \mathrm{yr}$, respectively) and BMI $\left(49.5 \pm 1.0\right.$ vs $\left.48.9 \pm 1.1 \mathrm{~kg} / \mathrm{m}^{2}\right)$. Groups differed significantly ( $\mathrm{P}=$ 0.004 ) only on triglyceride levels, with higher values in BED participants. In contrast to the surgery groups, participants with BED who were treated by surgery versus lifestyle 
modification differed significantly on several variables. The Lifestyle-BED group, compared to Surgery-BED, had a significantly greater percentage of African Americans (53.1 vs $22.2 \% ; \mathrm{P}=0.023)$, as well as a significantly lower baseline BMI (44.3 \pm 0.7 vs $48.9 \pm 1.1$; $\mathrm{P}$ $<0.001)$. The two groups also differed significantly on baseline triglycerides $(P=0.008)$, glucose $(\mathrm{P}=0.010)$, and LDL cholesterol $(\mathrm{P}=0.009)$ (see Table 1$)$.

\section{Surgery Participants: Effects of BED on Weight Loss}

At month 12, among surgically-treated participants in the modified ITT population, those with preoperative BED lost $22.1 \pm 1.7 \%$ of initial weight, compared with $24.2 \pm 1.3 \%$ for those free of this disorder (see Figure 2). Weight losses of the two groups did not differ significantly at this time $(\mathrm{P}=0.309)$ or at months $2(\mathrm{P}=0.897)$ or $6(\mathrm{P}=0.790)$. There also were no significant differences between the two groups in the percentage of participants who achieved losses of $5 \%(\mathrm{P}=0.633), 10 \%(\mathrm{P}=0.552)$, or $20 \%(\mathrm{P}=0.231)$ of initial weight (see Table 2).

An exploratory analysis examined differences in weight loss between individuals who were diagnosed with binge eating at month 12 , regardless of whether this behavior had been present at baseline. Of the 30 participants who had BED at baseline and completed the 12month assessment, 5 were determined to still binge eat at this time (in the prior 28 days), compared to 1 participant in the Surgery-nonBED group (all of whom had been free of BED at baseline). No significant differences in weight loss were observed at month 12 between the 6 participants who engaged in postoperative binge eating and the 70 participants who were free of this behavior $(P=0.264)$. Mean losses for the two groups were $20.2 \pm 0.9 \%$ and $24.3 \pm 3.5 \%$, respectively.

Similar analyses revealed that the presence of subjective binge episodes (SBEs), whether present before surgery or at the 12-month evaluation, did not affect weight loss. At month 12 , for example, the 11 participants who reported two or more SBEs in the prior 28 days lost $21.8 \pm 2.5 \%$ of initial weight, compared with $24.6 \pm 1.0 \%$ in the 65 patients who did not report this behavior $(\mathrm{P}=0.314)$.

Changes in CVD risk factors-Table 2 shows that surgically-treated participants in the modified ITT population, with or without preoperative BED, achieved substantial decreases in several CVD risk factors at month 12, although there were no significant differences between groups. Collapsing across the two groups, triglycerides declined by $36.8 \pm 7.6 \mathrm{mg}$ / $\mathrm{dl}(\mathrm{P}<0.001)$, total cholesterol by $12.3 \pm 4.8 \mathrm{mg} / \mathrm{dl}(\mathrm{P}=0.003)$, and LDL cholesterol by $14.9 \pm 4.5 \mathrm{mg} / \mathrm{dl}(\mathrm{P}<0.001)$. HDL cholesterol increased by $9.3 \pm 1.9 \mathrm{mg} / \mathrm{dl}(\mathrm{P}<0.001)$. Glucose and insulin declined by $17.6 \pm 3.0 \mathrm{mg} / \mathrm{dl}(\mathrm{P}<0.001)$ and $12.4 \pm 2.9 \mu \mathrm{U} / \mathrm{mL}(\mathrm{p}<$ 0.001 ), respectively, yielding a decline in HOMA of $3.4 \pm 0.7$ ( $\mathrm{P}<0.001)$; hs-CRP declined by $5.8 \pm 1.5 \mathrm{mg} / \mathrm{L}(\mathrm{P}<0.001)$.

Factors affecting weight loss in surgery participants-Of the co-variates included in the statistical analyses, type of surgery was most strongly related to weight loss at month 12. The 59 participants in the modified ITT population who received RYGB lost $29.9 \pm$ $1.7 \%$ of initial weight, compared to $12.9 \pm 1.9 \%$ in the 36 participants who underwent LAGB $(\mathrm{P}<0.001)$. (Equal percentages of patients in the BED and non-BED groups had RYGB -- 58.3\% vs $64.4 \% ; \mathrm{P}=0.664$.) Non-Hispanic whites lost more weight than members of ethnic minorities $(24.8 \pm 1.2 \%$ vs $20.1 \pm 1.7 \%$ of initial weight; $\mathrm{P}=0.012)$. Age, gender, baseline BMI, and preoperative diabetes status were not significantly related to weight loss. 


\section{BED Participants: Comparison of Lifestyle Modification and Surgery}

Participants with BED who received lifestyle modification lost $10.3 \pm 1.5 \%$ of initial weight at month 12 , compared with the previously described $22.1 \pm 1.7 \%$ for BED participants who had surgery. Differences in weight loss between groups (for the modified ITT population) were significant at all times (all Ps $<0.001$ ). At month 12 , a significantly greater percentage of BED participants treated by surgery than by lifestyle modification lost $\geq 5 \%(P=0.004)$, $\geq 10 \%$ ( $P<0.001$ ), and $\geq 20 \%$ ( $<<0.001$ ) of initial weight (see Table 2$)$.

Binge eating - The number of binge eating days declined sharply in both groups during the first 6 months, as shown in Figure 3A. In the lifestyle modification group, the mean number of days (as assessed for the prior 28 days) fell from $13.0 \pm 0.9$ to $0 \pm 0.8$ days at month 6 , as compared to a decline from $9.5 \pm 1.2$ to $0 \pm 0.9$ days in surgery participants. At month 12 , however, the two groups increased to $4.0 \pm 1.1$ and $1.5 \pm 1.3$ days, respectively. There were no significant differences between groups in changes in binge days at any time (all Ps > 0.180). Similarly, there were no significant differences between the two groups at any time in the remission of BED (all Ps > 0.458). At month 6, 94.4\% (34 of 36) of participants treated by surgery no longer met criteria for BED (for the prior 3 months), compared with $87.8 \%$ (43 of 49) of those who received lifestyle modification.

Corresponding values at month 12 were $91.7 \%$ (33 of 36) and $85.7 \%$ (42 of 49), respectively.

Figure 3B shows that the mean number of subjective binge eating days was relatively low in both groups at baseline and remained so throughout the year. There also were no significant differences between groups at any time on changes on this measure ( $\mathrm{Ps}>0.875$ ).

CVD risk factors-As shown in Table 2, surgically treated participants with BED tended to achieve greater reductions in CVD risk factors at month 12 than did BED participants who received lifestyle modification. The greatest difference was in the decline in triglycerides ( $39.6 \pm 8.2$ vs $17.8 \pm 6.4 \mathrm{mg} / \mathrm{dl}$, respectively). However, none of the differences between groups reached the significance level (i.e., $\mathrm{P} \leq 0.01$ ) chosen to control for multiple comparisons.

\section{Discussion}

This study's principal finding was that the preoperative presence of BED, as defined by proposed DSM-5 criteria, did not significantly attenuate weight loss at 1 year following bariatric surgery. Patients with BED lost $22.1 \%$ of initial weight, compared to $24.2 \%$ for those without this disorder, and were equally successful in achieving all categorical weight losses (i.e., $\geq 5 \%, 10 \%$, and $20 \%$ ). Weight losses in both surgery groups were associated with clinically significant improvements in cardiovascular risk factors including triglycerides, LDL and HDL cholesterol, and hs-CRP. Improvements in glycemic control and insulin sensitivity also were observed. There were no statistically significant differences between groups on any of these measures.

The present findings add to several recent reports which found that preoperative BED (or sub-clinical BED) did not impair weight loss following bariatric surgery (17-21). The strength of the current study resides in its use of the EDE to diagnose BED preoperatively and its inclusion of a comparison group of individuals who were free of a current eating disorder, thus, encouraging maximum separation between groups. (Participants judged to be free of a current eating disorder did not engage in more than one objective overeating episode per week for the prior 3 months, and a majority did not report any such episodes.) Prior studies which reported that preoperative BED attenuated weight loss either assessed this condition using self-report questionnaires or diagnosed BED retrospectively. Both 
approaches have significant limitations. On the basis of the present findings, and those of others (17-21), we do not believe that the preoperative presence of BED, by itself, should be cause to defer patients from bariatric surgery.

This study included a non-randomized treatment comparison group, comprised of participants who were diagnosed with BED and met the BMI criterion for bariatric surgery but sought weight loss by lifestyle modification. These participants were similar to those with BED who underwent bariatric surgery, although the former group did have a significantly lower BMI and included a significantly greater percentage of AfricanAmericans. The comprehensive lifestyle intervention produced a mean loss of $10.3 \%$ of initial weight at 1 year, which was comparable to losses reported at 1 year by Ryan et al (47) in participants who received lifestyle modification combined with meal replacements and weight loss medication.

Caution is required in interpreting the results for participants with BED who received bariatric surgery versus lifestyle modification; the two groups may have differed in important ways, beyond their motivation for their respective treatments. Nonetheless, participants with preoperative BED who underwent bariatric surgery lost more than twice as much weight at 1 year as those treated by lifestyle modification. In addition, participants in the former group had greater improvements in levels of triglycerides and HDL cholesterol, although differences between groups did not reach pre-specified $\mathrm{P}$ values (i.e., $\mathrm{P} \leq 0.01$ ). Participants in both groups experienced marked reductions in their number of binge eating days. Objective binge eating episodes were virtually eliminated during the first 6 months but increased slightly in both groups at 1 year. A randomized controlled trial would be needed to confirm the apparent superiority of bariatric surgery over lifestyle modification in obese individuals with BED. Such a trial likely would present challenges in identifying participants who would be willing (and able, based potentially on insurance coverage) to accept assignment to both groups. In addition, participants would need to be followed longer determine whether the differences between groups that we observed at 1 year would be maintained. In such a trial, conducted in patients without BED, O'Brien et al (48) found that the greater weight loss achieved at 1 year with laparoscopic adjustable gastric banding versus lifestyle modification increased further at 2 years.

In addition to its strengths, the present investigation had a number of limitations, including the lack of a randomized design and the inability to obtain any post-baseline weights on 36 of 131 patients who completed the EDE and proceeded to have surgery. The great majority of these non-participants either did not respond to repeated efforts to contact them or informed us that they no longer had time to attend study visits. These non-participants were significantly younger than surgically-treated participants who provided a post-baseline measure of weight but otherwise did not differ on any of the other baseline demographic variables examined. In addition, approximately equal numbers of patients with and without BED (29.4\% and $26.2 \%$, respectively) failed to complete at least one post-surgical assessment. We do not know how the weight losses of these 36 non-participants would have compared with those of the 131 surgery patients who participated in follow-up assessments. In contrast to surgically-treated individuals, only $3.9 \%$ of participants in the Lifestyle Modification intervention failed to provide a post-baseline measurement of weight. Their greater participation in the assessments likely reflected their having received weekly and then bi-weekly weight reduction therapy from our research team for the first 40 weeks, which we think strengthened their commitment to participating in the outcomes assessments.

We believe that examination of our modified-ITT population, as commonly used in trials of weight loss medications $(49,50)$, was the most appropriate method of handling the surgical participants who did not provide any post-baseline measurements. For all participants who 
provided at least one post-baseline measurement of weight, the use of the mixed-effects model allowed us to estimate weight losses at month 12 for any participant who did not provide a measured weight at this time.

We note that our study's principal conclusion - that the preoperative presence of BED did not significantly reduce 1-year weight loss following surgery - applies to individuals who were diagnosed with BED according to proposed DSM 5 criteria (i.e., one binge episode a week rather than two, as required by DSM-IV). It is possible that a more frequent rate of binge eating than examined in this study would have been associated with suboptimal weight loss, although a recent investigation that included such a comparison did not observe smaller weight losses in persons with more frequent preoperative binge eating (20). We also note that BED, as determined using the EDE or other investigator-driven assessments, was uncommon in our bariatric surgery population. Our prior study of 210 consecutive surgery applicants revealed that only $4.2 \%$ were diagnosed with BED, using DSM-IV criteria, and only a total of 5.6\% met criteria when the required frequency of binge eating was reduced to once per week (10). (By contrast, based on patients' self-reports of binge eating, 15.7\% met DSM-IV criteria for BED, and 19.6\% met proposed DSM-5 criteria.) For the present study, we screened $>1,200$ surgery candidates to obtain the 62 who agreed to participate in the study and had BED, as determined by DSM 5 criteria.

We found no evidence in this study that the presence, at 1 year, of either objective or subjective binge eating was associated with significantly smaller weight loss at this time. However, other investigators $(20,21)$ have reported that loss of control over eating following surgery, as exemplified by subjective binge episodes, is associated with suboptimal longterm (> 1 year) weight loss. White et al (20), for example, found that patients who reported loss of control over eating at 12 months post-surgery lost significantly less weight at month 24 than did patients who were free of this condition. By contrast, preoperative reports of loss of control did not predict weight loss at any time following surgery. Sarwer et al (51) similarly found that poor self-reported dietary adherence 20 weeks postsurgery, which may have reflected loss of control over eating, was associated with diminished weight loss at week 92. Collectively, these findings suggest that periodic monitoring of eating behavior could be useful in patients with sub-optimal weight loss at milestone visits at $6,12,18$, and 24 months post-surgery. Patients who reported BED or other forms of loss of control over eating post-surgery (regardless of its presence or absence before surgery) could potentially benefit from cognitive behavioral intervention (16). The present 1-year study may not have been sufficiently long to detect the emergence and consequences of loss of control over eating.

In summary, the present study found that patients with BED who underwent bariatric surgery achieved 1-year weight losses and improvements in CVD risk factors comparable to those of individuals without BED. Moreover, in patients with BED, surgical intervention was associated with significantly greater weight loss than lifestyle modification and with comparable improvements in eating behavior and CVD risk factors. Based on these and other results (17-21), we did not believe that preoperative BED, by itself, provides reason for practitioners to defer severely obese patients from bariatric surgery.

\section{Acknowledgments}

Preparation of this manuscript was supported, in part, by National Institutes of Health grants DK069652 and DK065018.

The authors gratefully acknowledge Health Management Resources for supplying meal replacements for this study, as well as Rebecca Stack, Faith Cottrell, and Louis Bergelson for their many contributions in coordinating the recruitment, assessment, and treatment of study participants. 


\section{References}

1. American Psychiatric Association. Diagnostic and Statistical Manual of Mental Disorders. Washington, DC: 2000. (4th ed. TR)

2. Spitzer RL, Devlin M, Walsh TB, et al. Binge eating disorder: A multisite field trial of the diagnostic criteria. Int J Eat Disord. 1992; 11:191-203.

3. Kalarchian MA, Marcus MD, Levine MD, et al. Psychiatric disorders among bariatric surgery candidates: Relationship to obesity and functional health status. Am J Psychiatry. 2007; 164:328334. [PubMed: 17267797]

4. Wadden TA, Sarwer DB, Fabricatore AN, Jones L, Stack R, Williams NS. Psychosocial and behavioral status of patients undergoing bariatric surgery: what to expect before and after surgery. Med Clin North Am. 2007; 9:451-469. [PubMed: 17509389]

5. Sarwer DB, Wadden TA, Fabricatore AN. Psychosocial and behavioral aspects of bariatric surgery. Obes Res. 2005; 13:639-648. [PubMed: 15897471]

6. Jones-Corneille LR, Wadden TA, Sarwer DB, et al. Axis I psychopathology in bariatric surgery candidates with and without binge eating disorder: results of structured clinical interviews. Obesity Surg. in press.

7. Zimmerman M, Francione-Witt C, Chelminski I, et al. Presurgical psychiatric evaluations of candidates for bariatric surgery, part 1: reliability and reasons for and frequency of exclusion. J Clin Psychiatry. 2007; 68:1557-1562. [PubMed: 17960972]

8. Kalarchian MA, Wilson GT, Brolin RE, Bradley L. Binge eating in bariatric surgery patients. Int J Eat Disord. 1998; 23:89-92. [PubMed: 9429923]

9. Dymek-Valentine M, Rienecke-Hoste R, Alverdy J. Assessment of binge eating disorder in morbidly obese patients evaluated for gastric bypass: SCID versus QEWP-R. Eat Weight Disord. 2004; 9:211-216. [PubMed: 15656016]

10. Allison KC, Wadden TA, Sarwer DB, et al. Night eating syndrome and binge eating disorder among persons seeking bariatric surgery: prevalence and related features. Obesity. 2006; 14 Suppl 2:77S-82S. [PubMed: 16648598]

11. Adami GF, Gandolfo P, Bauer B, et al. Binge eating in massively obese patients undergoing bariatric surgery. Int J Eat Disord. 1995; 17:45-50. [PubMed: 7894452]

12. Hsu LK, Sullivan SP, Benotti PN. Eating disturbances and outcome of gastric bypass surgery: a pilot study. Int J Eat Disord. 1997; 21:385-390. [PubMed: 9138051]

13. de Zwaan M, Lancaster KL, Mitchell JE, et al. Health-related quality of life in morbidly Healthrelated quality of life in morbidly. Obes Surg. 2002; 12:773-780. [PubMed: 12568181]

14. Pekkarinen T, Koskela K, Huikuri K, Mustajoki P. Long-term results of gastroplasty for morbid obesity: binge-eating as a predictor of poor outcome. Obes Surg. 1994; 4:248-255. [PubMed: 10742782]

15. Sallet PC, Sallet JA, Dixon JB, et al. Eating behavior as a prognostic factor for weight loss after gastric bypass. Obes Surg. 2007; 17:445-451. [PubMed: 17608254]

16. Ashton K, Drerup M, Windover A, Heinberg L. Brief, four-session group CBT reduces binge eating behaviors among bariatric surgery candidates. Surg Obes Relat Dis. 2009; 5:257-262. [PubMed: 19250884]

17. Alger-Mayer S, Rosati C, Polimeni JM, Malone M. Preoperative binge eating status and gastric bypass surgery: a long-term outcome study. Obes Surg. 2009; 19:139-145. [PubMed: 18478306]

18. Fujioka K, Yan E, Wang HJ, Li Z. Evaluating preoperative weight loss, binge eating disorder, and sexual abuse history on Roux-en-Y gastric bypass outcome. Surg Obes Relat Dis. 2008; 4:137143. [PubMed: 18359454]

19. White MA, Masheb RM, Rothschild BS, Burke-Martindale CH, Grilo CM. The prognostic significance of regular binge eating in extremely obese gastric bypass patients: 12-month postoperative outcomes. J Clin Psychiatry. 2006; 67:1928-1935. [PubMed: 17194271]

20. White MA, Kalarchian MA, Masheb RM, Marcus MD, Grilo CM. Loss of control over eating predicts outcomes in bariatric surgery patients: a prospective, 24-month follow-up study. J Clin Psychiatry. 2010; 71:175-184. [PubMed: 19852902] 
21. Burgmer R, Grigutsch K, Zipfel S, et al. The influence of eating behavior and eating pathology on weight loss after gastric restriction operations. Obes Surg. 2005; 15:684-691. [PubMed: 15946461]

22. Kalarchian MA, Marcus MD, Wilson GT, Labouvie EW, Brolin RE, LaMarca LB. Binge eating among gastric bypass patients at long-term follow-up. Obes Surg. 2002; 12:270-275. [PubMed: 11975227]

23. Colles SL, Dixon JB, O'Brien PE. Grazing and loss of control related to eating: two high-risk factors following bariatric surgery. Obesity. 2008; 16:615-622. [PubMed: 18239603]

24. Boan J, Kolotkin RL, Westman EC, McMahon RL, Grant JP. Binge eating, quality of life and physical activity improve after Roux-en-Y gastric bypass for morbid obesity. Obes Surg. 2004; 14:341-348. [PubMed: 15072655]

25. Kalarchian MA, Wilson GT, Brolin RE, Bradley L. Effects of bariatric surgery on binge eating and related psychopathology. Eat Weight Disord. 1999; 4:1-5. [PubMed: 10728171]

26. Mechanick JI, Kushner RF, Sugerman HJ, et al. American Association of Clinical Endocrinologists, The Obesity Society, and American Society for Metabolic \& Bariatric Surgery medical guidelines for clinical practice for the perioperative nutritional, metabolic, and nonsurgical support of the bariatric surgery patient. Obesity. 2009; 17 Suppl 1:S1-S70. v. [PubMed: 19319140]

27. Sarwer DB. Comment on: brief, four session group CBT reduces binge eating behaviors among bariatric surgery candidates (Ashton K, et al. 2009;5:257-62). Surg Obes Relat Dis. 2009; 5:407408. [PubMed: 19356996]

28. Fairburn, CG.; Cooper, Z. The eating disorder examination. In: Fairburn, CG.; Wilson, GT., editors. Binge Eating: Nature, Assessment, and Treatment. 12th ed.. New York: Guilford Press; 1993. p. 317-360.

29. Wadden TA, Sarwer DB. Behavioral assessment of candidates for bariatric surgery: a patientoriented approach. Obesity. 2006; 14 Suppl 2:53S-62S. [PubMed: 16648595]

30. Beck, AT.; Steer, RA.; Brown, GK. Beck Depression Inventory II (BDI-II) Manual. San Antonio, TX: Harcourt Brace \& Company; 1993.

31. Wadden TA, Foster GD. Weight and lifestyle inventory. Obesity. 2006; 14 Suppl 2:99S-118S. [PubMed: 16648601]

32. Spitzer RL, Devlin M, Walsh TB, et al. Binge eating disorder: a multisite field trial of the diagnostic criteria. Int J Eat Disord. 1992; 11:191-203.

33. Cooper Z, Cooper P, Fairburn CG. The validity of the Eating Disorder Examination and its subscales. Brit J Psychiatry. 1989; 154:807-812. [PubMed: 2597887]

34. Wilson GT, Wilfley DE, Agras WS, Bryson SW. Psychological treatments of binge eating disorder. Arch Gen Psychiatry. 2010; 67:94-101. [PubMed: 20048227]

35. American Psychiatric Association. DSM-5 Proposed Diagnostic Criteria for Binge Eating Disorder. [Accessed April 22, 2010]. http://www.dsm5.org/ProposedRevisions/Pages/proposedrevision.aspx?rid=372.

36. Wilson GT, Sysko R. Frequency of binge eating episodes in bulimia nervosa and binge eating disorder: Diagnostic considerations. Int J Eat Disord. 2009; 42:603-610. [PubMed: 19610014]

37. Elder KA, Grilo CM, Masheb RM, et al. Comparison of two self-report instruments for assessing binge eating in bariatric surgery candidates. Behav Ther. 2006; 44:545-560.

38. Striegel-Moore RH, Dohm FA, Solomon EE, et al. Subthreshold binge eating disorder. Int J Eat Disord. 2000; 27:270-278. [PubMed: 10694712]

39. Buchwald H, Avidor Y, Braunwald E, et al. Bariatric surgery: a systematic review and metaanalysis. JAMA. 2004; 292:1724-1737. [PubMed: 15479938]

40. Tice JA, Karliner L, Walsh J, Petersen AJ, Feldman MD. Gastric banding or bypass? A systematic review comparing the two most popular bariatric procedures. Am J Med. 2008; 121:885-893. [PubMed: 18823860]

41. Wadden TA, Berkowitz RI, Womble LG, et al. Randomized trial of lifestyle modification and pharmacotherapy for obesity. N Engl J Med. 2005; 353:2111-2120. [PubMed: 16291981] 
42. Wadden TA, Berkowitz RI, Sarwer DB, et al. Benefits of lifestyle modification in the pharmacologic treatment of obesity: a randomized trial. Arch Intern Med. 2001; 161:218-227. [PubMed: 11176735]

43. Wadden TA, Foster GD, Sarwer DB, et al. Dieting and the development of eating disorders in obese women: results of a randomized controlled trial. Am J Clin Nutr. 2004; 80:560-568. [PubMed: 15321793]

44. Flechtner-Mors M, Ditschuneit HH, Johnson TD, Suchard MA, Adler G. Metabolic and weight loss effects of long-term dietary intervention in obese patients: four-year results. Obes Res. 2000; 8:399-402. [PubMed: 10968732]

45. The Look AHEAD Research Group. Long term effects of lifestyle intervention on weight and cardiovascular risk factors in individuals with type 2 diabetes: four year results of the Look AHEAD trial. Arch Intern Med. 2010; 170:1566-1575. [PubMed: 20876408]

46. Wilson GT. Psychological treatment of eating disorders. Annu Rev Clin Psychol. 2005; 1:439-465. [PubMed: 17716095]

47. Ryan DH, Johnson WD, Myers VH, et al. Nonsurgical weight loss for extreme obesity in primary care settings: results of the Louisiana Obese Subjects Study. Arch Intern Med. 2010; 170:146-154. [PubMed: 20101009]

48. O'Brien PE, Dixon JB, Laurie C, et al. Treatment of mild to moderate obesity with laparoscopic adjustable gastric banding or an intensive medical program: a randomized trial. Ann Intern Med. 2006; 144:625-633. [PubMed: 16670131]

49. Pi-Sunyer FX, Aronne LJ, Heshmati HM, et al. Effect of rimonabant, a cannabinoid-1 receptor blocker, on weight and cardiometabolic risk factors in overweight or obese patients: RIO-North America: a randomized controlled trial. JAMA. 2006; 295:761-775. [PubMed: 16478899]

50. Greenway FL, Fujioka K, Plodkowski RA, et al. Effect of naltrexone plus bupropion on weight loss in overweight and obese adults (COR-I): a multicentre, randomised, double-blind, placebocontrolled, phase 3 trial. Lancet. 2010; 376:595-605. [PubMed: 20673995]

51. Sarwer DB, Wadden TA, Moore RH, et al. Preoperative eating behavior, postoperative dietary adherence, and weight loss after gastric bypass surgery. Surg Obes Relat Dis. 2008; 4:640-646. [PubMed: 18586571] 


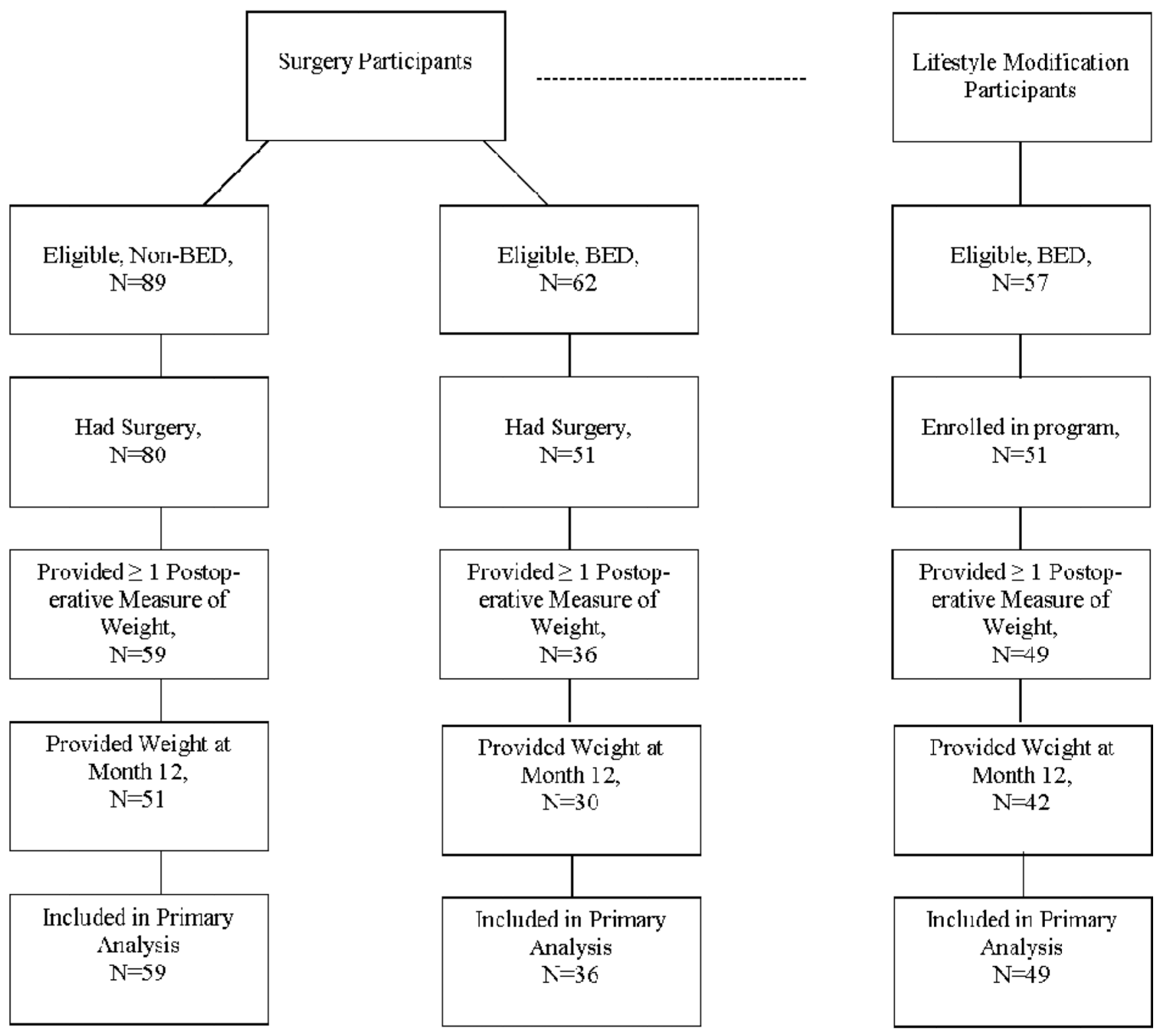

Figure 1.

Recruitment and retention of participants in the three observational groups. Two groups were selected to have binge eating disorder (BED) and one to be free of this condition (nonBED). Two groups of participants had surgery and the third received lifestyle modification. 


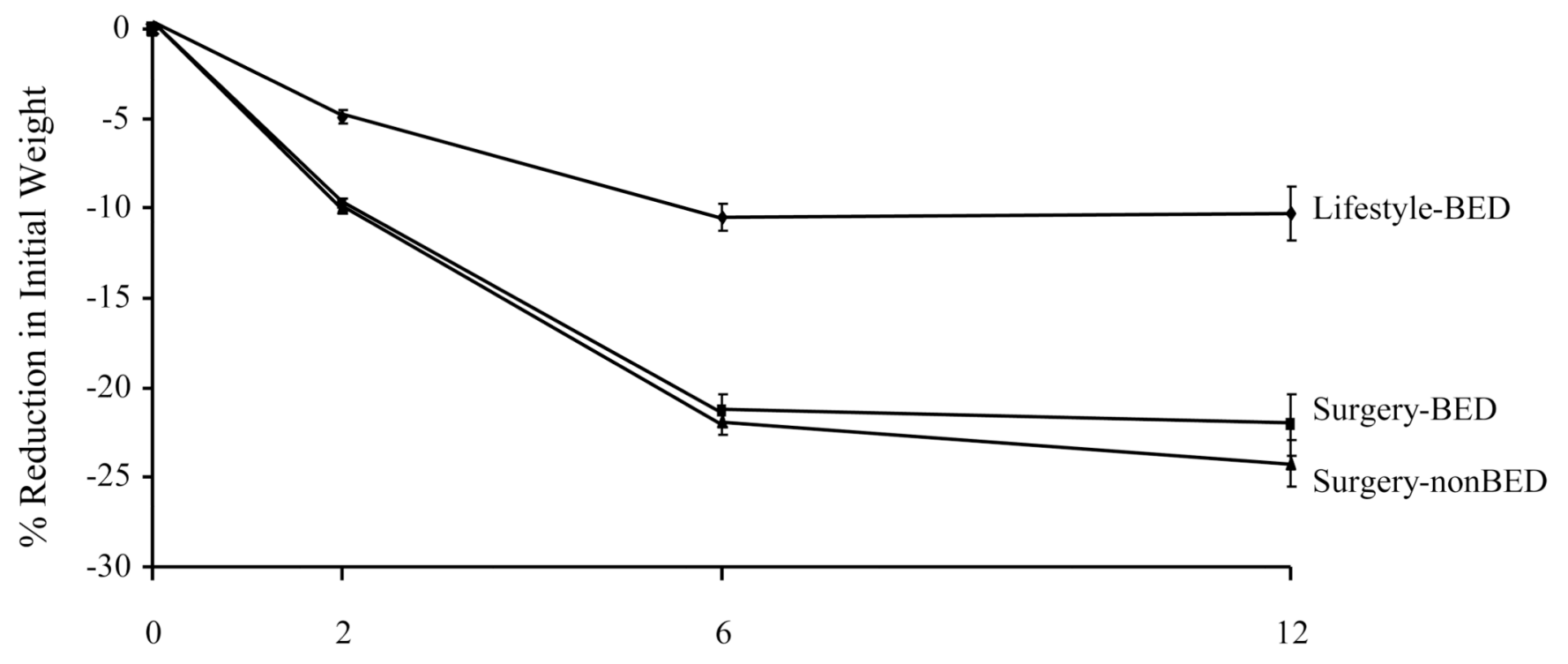

Months

Figure 2.

Mean 12-month weight losses of participants in the three observational groups. Losses of patients with binge eating disorder (BED) who received surgery (BED-Surgery) did not differ significantly from those of participants who were free of BED and also had surgery (nonBED-Surgery). BED-Surgery participants lost significantly $(\mathrm{p}<0.001)$ more weight at month 12 than did participants with BED treated by lifestyle modification (BED-Lifestyle). Analyses were conducted controlling for initial BMI, type of surgery, age, ethnicity, and presence of diabetes. 


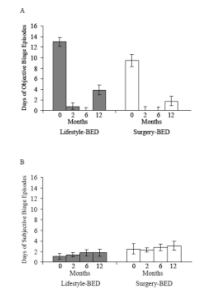

Figure 3.

A. Changes in objective binge eating days in participants with BED who received surgery (BED-Surgery) and lifestyle modification (BED-Lifestyle). There were no significant differences between these two groups at any time. The mean values for participants who were free of BED and had surgery (nonBED-Surgery) were close to 0 at all times.

B. Changes in subjective binge eating days in participants with BED who received surgery (BED-Surgery) and lifestyle modification (BED-Lifestyle). There were no significant differences between these two groups at any time. The mean values for participants who were free of BED and had surgery (nonBED-Surgery) were close to 0 at all times. 
Table 1

Participants' baseline characteristics

\begin{tabular}{|c|c|c|c|}
\hline Characteristic & $\begin{array}{l}\text { Surgery-nonBED } \\
\qquad(\mathrm{N}=\mathbf{5 9})\end{array}$ & $\begin{array}{l}\text { Surgery-BED } \\
\qquad(\mathbf{N}=\mathbf{3 6})\end{array}$ & $\begin{array}{l}\text { Lifestyle-BED } \\
\qquad(\mathrm{N}=49)\end{array}$ \\
\hline \multicolumn{4}{|l|}{ Sex, N (\%) } \\
\hline Female & $49(83.1 \%)$ & $26(72.2 \%)$ & $39(79.6 \%)$ \\
\hline Male & $10(16.9 \%)$ & $10(27.8 \%)$ & $10(20.4 \%)$ \\
\hline \multicolumn{4}{|l|}{ Race/Ethnicity, N (\%) } \\
\hline Black/African American & $14(23.7 \%)$ & $8(22.2 \%)$ & $26(53.1 \%)$ \\
\hline Hispanic & $3(5.1 \%)$ & $1(2.8 \%)$ & $3(6.1 \%)$ \\
\hline Non-Hispanic White & $42(71.2 \%)$ & $27(75.0 \%)$ & $18(36.7 \%)$ \\
\hline Other & $0(0 \%)$ & $0(0 \%)$ & $2(4.1 \%)$ \\
\hline \multicolumn{4}{|l|}{ Education N (\%) } \\
\hline$<12^{\text {th }}$ grade & $2(3.4 \%)$ & $1(2.8 \%)$ & $3(6.1 \%)$ \\
\hline High school/G.E.D. & $34(57.6 \%)$ & $19(52.8 \%)$ & $26(53.1 \%)$ \\
\hline Bachelors degree & $13(22.0 \%)$ & $9(25.0 \%)$ & $14(28.6 \%)$ \\
\hline Graduate or professional degree & $10(16.9 \%)$ & $7(19.4 \%)$ & $6(12.2 \%)$ \\
\hline Age (yr) & $43.8 \pm 1.3$ & $47.0 \pm 1.6$ & $43.8 \pm 1.4$ \\
\hline Weight (kg) & $139.3 \pm 3.7$ & $138.7 \pm 4.0$ & $125.8 \pm 2.9$ \\
\hline Height (cm) & $167.3 \pm 1.1$ & $168.8 \pm 1.5$ & $168.2 \pm 1.2$ \\
\hline BMI $\left(\mathrm{kg} / \mathrm{m}^{2}\right)$ & $49.5 \pm 1.0$ & $48.9 \pm 1.1$ & $44.3 \pm 0.7$ \\
\hline Triglycerides (mg/dl) & $128.6 \pm 8.7$ & $176.0 \pm 15.0$ & $125.5 \pm 11.4$ \\
\hline \multicolumn{4}{|l|}{ Cholesterol } \\
\hline Total (mg/dl) & $178.2 \pm 5.1$ & $180.7 \pm 7.4$ & $194.9 \pm 5.0$ \\
\hline LDL-C (mg/dl) & $102.0 \pm 4.2$ & $97.6 \pm 7.2$ & $119.2 \pm 4.4$ \\
\hline HDL-C (mg/dl) & $49.4 \pm 1.7$ & $47.9 \pm 2.4$ & $50.7 \pm 2.2$ \\
\hline hs-CRP (mg/L) & $15.6 \pm 2.0$ & $10.3 \pm 2.1$ & $9.9 \pm 1.3$ \\
\hline Glucose (mg/dl) & $107.7 \pm 3.3$ & $118.3 \pm 6.7$ & $100.8 \pm 2.8$ \\
\hline Insulin $(\mu \mathrm{U} / \mathrm{ml})$ & $18.4 \pm 1.9$ & $28.0 \pm 7.4$ & $15.7 \pm 1.4$ \\
\hline HOMA & $5.1 \pm 0.7$ & $5.1 \pm 0.7$ & $4.2 \pm 0.5$ \\
\hline Type 2 diabetes, $\mathrm{N}(\%)$ & $14(23.7 \%)$ & $11(30.6 \%)$ & $5(10.2 \%)$ \\
\hline
\end{tabular}

Note: Values shown are mean $\pm \mathrm{SE}$. $\mathrm{LDL}=$ low density lipoprotein; $\mathrm{HDL}=$ high density lipoprotein; hs-CRP= high sensitivity $\mathrm{C}$ reactive protein; HOMA $=$ homeostasis method of insulin resistance. Statistically significant differences between groups are described in the text. 
Table 2

Number (\%) of participants in each of the three interventions ho lost $\geq 5 \%, \geq 10 \%$, and $\geq 20 \%$ of initial weight at 1 year.

\begin{tabular}{ll}
\hline Weight Loss Criterion & Month 12 \\
\hline$\geq 5 \%$ Loss & \\
Non-BED Surgery & $48(81.4 \%)$ \\
BED-Surgery & $27(75.0 \%)$ \\
BED-Lifestyle & $20(40.8 \%)$ \\
$\geq 10 \%$ Loss & \\
Non-BED Surgery & $44(74.6 \%)$ \\
BED-Surgery & $24(66.7 \%)$ \\
BED-Lifestyle & $13(26.5 \%)$ \\
$\geq 20 \%$ Loss & \\
Non-BED Surgery & $35(59.3 \%)$ \\
BED-Surgery & $16(44.4 \%)$ \\
BED-Lifestyle & $6(12.2 \%)$ \\
\hline
\end{tabular}

Note: The proportion of participants in the BED-Surgery group who achieved a $\geq 5 \%, \geq 10 \%$, and $\geq 20 \%$ weight loss was equivalent to the proportion of participants achieving the same level of weight loss in the Non-BED Surgery group, and significantly greater than the proportion of participants achieving the same level of weight loss in the BED-Lifestyle group (95\% CI). Statistically significant differences between groups are described in the text. 


\section{Table 3}

Changes at 1 year in weight and CVD risk factors in the three intervention groups.

\begin{tabular}{lccc}
\hline Variable & Surgery-nonBED & Surgery-BED & Lifestyle-BED \\
\hline Weight $(\mathrm{kg})$ & $-33.3 \pm 1.7$ & $-29.2 \pm 2.2$ & $-15.6 \pm 2.0$ \\
$\%$ change & $-24.2 \pm 1.3 \%$ & $-22.1 \pm 1.7 \%$ & $-10.3 \pm 1.5 \%$ \\
BMI $\left(\mathrm{kg} / \mathrm{m}^{2}\right)$ & $-11.8 \pm 0.6$ & $-10.9 \pm 0.8$ & $-5.6 \pm 0.7$ \\
Triglycerides $(\mathrm{mg} / \mathrm{dl})$ & $-35.1 \pm 7.3$ & $-39.6 \pm 8.2$ & $-17.7 \pm 6.4$ \\
Cholesterol & & & \\
Total $(\mathrm{mg} / \mathrm{dl})$ & $-11.8 \pm 4.6$ & $-13.0 \pm 5.2$ & $-3.1 \pm 4.5$ \\
LDL-C $(\mathrm{mg} / \mathrm{dl})$ & $-16.6 \pm 4.2$ & $-12.1 \pm 4.9$ & $-2.3 \pm 4.2$ \\
HDL-C $(\mathrm{mg} / \mathrm{dl})$ & $9.9 \pm 1.8$ & $8.4 \pm 2.1$ & $3.4 \pm 1.8$ \\
hs-CRP $(\mathrm{mg} / \mathrm{L})$ & $-6.7 \pm 1.4$ & $-4.3 \pm 1.6$ & $-5.5 \pm 1.5$ \\
Glucose $(\mathrm{mg} / \mathrm{dl})$ & $-19.0 \pm 2.8$ & $-15.4 \pm 3.3$ & $-0.8 \pm 2.7$ \\
Insulin $(\mu \mathrm{U} / \mathrm{ml})$ & $-13.3 \pm 2.7$ & $-10.9 \pm 3.2$ & $-4.6 \pm 2.5$ \\
HOMA & $-3.7 \pm 0.4$ & $-3.0 \pm 0.5$ & $-2.8 \pm 0.4$ \\
\hline
\end{tabular}

Note: Values shown are mean $\pm \mathrm{SE}$. BMI= body mass index; $\mathrm{LDL}=$ low density lipoprotein; HDL= high density lipoprotein; hs-CRP= high sensitivity $\mathrm{C}$ reactive protein; HOMA= homeostasis method of insulin resistance. Statistically significant differences between groups are described in the text. 